\title{
Palliative Langzeitbetreuung bei hirnorganischen Veränderungen - Herausforderungen und Chancen
}

\author{
Christel Nigg \\ Klinik Susenberg, Zürich
}

Wie im Artikel von Hedbom und Catschegn sehr anschaulich an zwei Patientenbeispielen verdeutlicht wird, spielen hirnorganische Veränderungen am Lebensende eine nicht zu unterschätzende Rolle. Neben den anderen belastenden Symptomen wie Schmerz, Übelkeit, Dyspnoe, Müdigkeit und Gewichtsverlust, um nur die häufigsten zu nennen, sind die Symptome im Rahmen der krankheitsassoziierten Persönlichkeitsveränderungen häufig therapeutisch herausfordernd. Auch für die Angehörigen, die in dieser Phase die Betroffenen unterstützen möchten, stellen diese Symptome eine grosse Belastung dar. Ein zusätzliches Problem entsteht dann, wenn Betroffene aufgrund der hirnorganischen Beeinträchtigung ihre Urteilsfähigkeit verlieren und Entscheidungen nur noch im Hinblick auf den mutmasslichen Willen getroffen werden können. Gerade bei Tumorerkrankungen, die im Verlauf eine Beteiligung des Gehirns vermuten lassen, sollten sehr früh die notwendigen administrativen Schritte wie Vorsorgeauftrag und Patientenverfügung unbedingt mit allen beteiligten Bezugspersonen besprochen und finalisiert werden.

Natürlich sind diese Themen belastend und schmerzhaft, aber häufig führt diese Auseinandersetzung mit der Endlichkeit nach Abschluss zu einer Entspannung in der Kommunikation und zu einer Beruhigung im Betreuungsnetz. Das Wissen, autonom und im Bewusstsein der Entscheidungsfähigkeit die Weichen zu stellen, ist für Patientinnen und Patienten wichtig. Sie wollen ja vor allem nicht belasten.

Viele Patientinnen und Patienten könnten, wenn die Infrastruktur vorhanden ist, auch zu Hause sterben. Beim Auftreten von Symptomen eines Delirs, einer Bewusstseinsveränderung, von Wahnvorstellungen und Verfolgungsideen kommt auch ein gut ausgebautes häusliches Betreuungsnetz rasch an seine Grenzen. Umso wichtiger ist in diesem Zusammenhang die rasche Verfügbarkeit von adäquaten Betreuungsstrukturen, wie sie eine spezielle Langzeitpalliativeinrichtung wie das Zürcher Lighthouse bieten kann. Ein klassisches Pflegeheim ist mit diesen Situationen häufig überfordert, sodass dann am Lebensende manchmal eine Einweisung in die Psychiatrie erfolgen muss. Dort ist das Manage- ment der somatischen Symptome je nach Verfügbarkeit von palliativmedizinisch erfahrenen Medizinerinnen und Medizinern schwierig umzusetzen, ganz zu schweigen von der umfassenden Betreuung von Menschen am Lebensende. Auch für die Pflegenden in der psychiatrischen Institution kann diese Situation belastend sein, wenn das Gefühl entsteht, der Patientin / dem Patienten und den Angehörigen nicht gerecht werden zu können. Ausserdem ist die Einweisung eines sterbenden Menschen in die Psychiatrie auch mit Scham für die Angehörigen besetzt. Solche Entwicklungen können mit einer gewissen Voraussicht vermieden werden.

Palliative Langzeitbetreuung muss im Gegensatz zur spezialisierten Palliative Care im Krankenhaus nicht von der Krankenkasse getragen werden. Dies ist ein klarer Missstand. Die Betroffenen sind nicht mehr akutspitalpflichtig, aber keine klassischen Pflegeheimpatientinnen/patienten, weil mehr medizinische Fachintervention erforderlich ist.

Im Hinblick darauf muss die Finanzierung dieser Versorgungsstrukturen überdacht werden, die weitgehend zu Lasten der Patientin/des Patienten bzw. der Familien gehen. Beim Vorliegen von «Institutionsbedürftigkeit» sollte hier ein alternatives Finanzierungsmodell zur Anwendung kommen, nicht zuletzt, um die notfallmässige Inanspruchnahme von Akutspitalstrukturen zu vermeiden.

\section{Bibliografie}

1. Hedbom S, Catschegn S: Fortgeschrittene Erkrankungen mit hirnorganischen Vernderungen. Praxis. 2020:109:1195-1199.

KD Dr. med. Christel Nigg

Klinik Susenberg

Chefärztin

Vorsitzende der Geschäftsleitung

Schreberweg 9

8044 Zürich

c.nigg@susenbergklinik.ch 